und überwachen. Der Lärmpegel ist deutlich geringer geworden, aber auch die Spannung, wenn man den Saal betritt. Die abgeschlossenen Geschäfte müssen sofort erfasst werden und den einzelnen Kontrahenten bestätigt werden. Die rechtlichen Regelungen für die Geschäfte werden in sogenannten Rahmenverträgen festgelegt. Diese Rahmenverträge sind überwiegend in englischer Sprache verfasst. Die ständige Weiterentwicklung der Geschäfte erfordert eine Abstimmung mit anderen Marktteilnehmern, da diese Geschäfte meist nicht durch eine Börse reguliert oder gesetzlich geregelt sind.

Ein Gremium für die Abstimmung ist der Arbeitskreis Finanztermingeschäfte des Bundesverbandes deutscher Banken, dem ich seit neun Jahren angehöre. Einmal im Quartal trifft man sich bei einer zum Arbeitskreis gehörenden Bank und bespricht aktuelle Themen und entwickelt standardisierte Verträge.

Diese Treffen und die gezielte Teilnahme an Seminaren sind wichtige Fortbildungen, aber auch eine angenehme Abwechslung zum Büroalltag. Voriges Jahr habe ich an einem Fachanwaltskurs für Bank- und Kapitalmarktrecht erfolgreich teilgenommen.

Dies erforderte - über einen Zeitraum von neun Monaten einmal im Monat drei Tage Lehrgang und anschließend das Lernen für die Klausuren. Wir Teilnehmer fühlten uns wieder in die Zeit der Staatsexamina versetzt.

Meine Kinder haben, seitdem ich bei einer Bank arbeite, Schulen mit amerikanischer Ausprägung besucht. Sie waren bis nachmittags in der Schule und konnten dort auch Mittag essen. Das hat mir die Organisation von Beruf und Familie sehr erleichtert. Inzwischen sind fast alle Kinder aus dem Haus. Jetzt kann ich abends mit einem ruhigeren Gewissen länger im Betrieb bleiben, was seit der Finanzkrise öfter notwendig ist. Nach meiner Erfahrung haben Vorgesetzte nur Verständnis für Frauen mit Familien, wenn sie selbst Kinder haben - egal ob Mann oder Frau.

Der Vorteil der Tätigkeit als Jurist(in) in der freien Wirtschaft besteht meines Erachtens darin, dass man bei der rechtsverbindlichen Umsetzung der Ideen der Kaufleute überwiegend gestalterisch tätig sein kann. Allerdings dauern die Abstimmungsprozesse länger, je größer die Unternehmen sind, wobei auch hierarchische Strukturen zu beachten sind. Die Erwartungshaltung bei einer Bank ist groß und der Zeitdruck speziell im Kapitalmarktbereich - enorm, da eine möglichst schnelle Umsetzung der Geschäfte entscheidend ist.

Dennoch arbeite ich gerne gerade in diesem Bereich, da ich hier, insbesondere durch die internationale Ausrichtung, meine amerikanisch-deutsche Prägung, meine Ausbildung und meinen beruflichen Werdegang nutzbringend einsetzen kann. Und dies, obwohl ich nicht von den horrenden Boni profitiere, die einige Banker erhalten.

Aber seien Sie versichert, auch hier ist ein Umdenken zumindest in Deutschland deutlich erkennbar.

\title{
Ein Dialog der Generationen - Hella Klose-Frantzen im Porträt
}

\section{Cindy Primus \\ Studentin, Brieselang \\ Olemia Flores Ramirez \\ Studentin, Berlin}

Hella Klose-Frantzen wurde am 2. Juni 1925 in Aachen als Tochter eines Kaufmanns geboren. Von ihm erbte sie auch ihren rationalen Verstand und ihre aufrechte Art. Der Vater war eine sehr wichtige Person in ihrem Leben, der sie förderte, aber auch forderte. So erhielt sie die Möglichkeit, 1944 das Gymnasium mit dem Abitur abzuschließen. Nach den Schrecken des 2. Weltkrieges und den Tätigkeiten in einer Munitionsfabrik und als Rotkreuzschwester begann sie das Studium der Rechtswissenschaften in Mainz (1946/47 bis 1951). „Damals war Jura noch ein Männerstudium. Wir waren gerade einmal zehn junge Frauen auf der Universität“, berichtete sie. Nach erfolgreicher Beendigung des Referendariats nahm Hella Klose-Frantzen zunächst eine Stelle als Regierungsassessorin in Aachen an. Sie entwickelte allerdings sehr schnell großes Interesse am Familienrecht und wechselte in das Anwaltsmetier. Ihre Neigung insbesondere zum Schei- dungsrecht begründete sich darauf, dass Frauen grundsätzlich im Rahmen einer Scheidung konsequent - sowohl rechtlich, als auch gesellschaftlich - benachteiligt wurden. Dies lief ihrem sehr ausgeprägten Gerechtigkeitssinn zuwider. Im Gespräch verriet sie aber auch noch, dass ein gewisser Hang zum Abenteuer und ihr Kampfgeist sie in Richtung ihres neuen Arbeitsfeldes führten. Es musste eine positive Veränderung für die Stellung der Frau im Scheidungsrecht erreicht werden und wer konnte dafür härter kämpfen als eine Frau. In dieser Zeit wurde auch ein neuer Name in die Mitgliederkartei des djb aufgenommen: Hella Klose-Frantzen. Die Gruppe in Aachen fasste damals nur sehr wenige Mitglieder, die sich allerdings - trotz ihrer geringen Anzahl - nicht minder stark für die Rechte der Frauen einsetzten. Hella KloseFrantzen hielt den Kontakt zu den Frauen des djb, soweit es ihr möglich war, obwohl sie neben dem „Vollzeitjob“ als Hausfrau und Mutter auch noch ihrem Beruf als Anwältin nachging. Diese Balance zwischen Beruf, Haushalt und Kindern war nicht immer leicht, allerdings hätte sie auch nicht ohne ihre Tätigkeit in der Anwaltschaft, die sie sehr liebte, leben können. Sie setzte sich sehr intensiv und leidenschaft- 
lich für ihre Mandantinnen im Rahmen des Familienrechts ein - und dies spürt man noch bis heute. Außerdem versuchte sie, die Entwicklung des Scheidungsrechts durch Vorträge und den daraus resultierenden inhaltlichen Diskurs vorwärts zu bringen. So lautete die Devise: Wenn man nicht darüber spricht und die Sache anpackt, wird sich niemals etwas ändern. Dieser Diskurs musste allerdings im Rahmen der Anwaltschaft geführt werden, da die meisten Richter zu dieser
Zeit Männer waren, die sich der Thematik des Scheidungsrechts größtenteils nicht widmen wollten.

So veränderten sich im Laufe der Zeit die Gesellschaft, das Scheidungsrecht und die Anzahl der Anwältinnen und Richterinnen. Und es wird sich weiterhin einiges ändern, wenn junge Frauen heutzutage auch der Devise folgen: Man muss die kritischen Themen selbstständig aufgreifen und bearbeiten, um Veränderung zu bewirken.

\section{Professorin und Mutter: Geht das zusammen?}

\section{Prof. Dr. Ute Sacksofsky, M.P.A. (Harvard) \\ Goethe-Universität, Frankfurt am Main}

Aufgewachsen bin ich in einer Kleinstadt im südlichen Schwarzwald, die mir in der Pubertät schnell zu klein wurde. So freute ich mich unheimlich auf das Studium - endlich raus. Ich begann mit dem Studium der Mathematik und Biologie für das Lehramt in Freiburg. Ausschlaggebend dafür war wohl die Begeisterung für eine phantastische Lehrerin mit genau dieser Fächerkombination und geringes Vorstellungsvermögen über denkbare Alternativen. Nach einem Semester musste ich aber einsehen, dass ich für Biologie denkbar ungeeignet war: Tiere und Pflanzen interessierten mich nicht wirklich (andere Mitstudierende standen begeistert früh auf, um Vögel zu beobachten - wäre mir nicht im Traum eingefallen), sezieren wollte ich Tiere schon gar nicht und Reagenzgläser tendierten dazu umzufallen, wenn ich in die Nähe kam. Die Mathematik machte mir zwar viel Spaß, aber niemand konnte mir erklären, was ich mit Mathematik einmal beruflich anfangen könnte (Informatik war in Freiburg noch nicht angekommen) außer dem Lehramt und das schied inzwischen aus; dem System Schule einmal entronnen, war mir klar geworden, dass ich dorthin auf keinen Fall zurückkehren wollte. Ein Studienfachwechsel stand an. In meinem ersten Semester hatte ich mir neben Mathematik und Biologie auch einige juristische Veranstaltungen angesehen und war sehr angetan. Jura schien mir die perfekte Verbindung von Logik, die meiner mathematischen Neigung entgegenkam, und Politik, die mich brennend interessierte.

Der Studienfachwechsel wurde mit einem Ortswechsel verbunden. Wegen einer engen Freundin und dem „linken Ruf“ wählte ich Marburg als Kontrastprogramm zum konservativen Freiburg. So kann man sich täuschen. Die Marburger Fakultät war erzkonservativ (z.B. wurde uns untersagt, Flugblätter im Hörsaalgebäude zu verteilen). Hinzu kam, dass viele der Lehrenden einfach schlecht waren. Sie indoktrinierten mit Mittelmaß und Mindermeinung. Nach einem Semester ging ich daher nach Freiburg zurück. Ich begriff schnell, dass mir Vorlesungen mit ihren Massen und dem bloßen Zuhören wenig brachten. Außerdem hatte ich dafür keine Zeit, weil ich mich in der Hochschulpolitik engagierte. Ich war über Jahre im Fachbe- reichsrat, in der Fachschaft und im AStA (bzw. u-asta - wir kämpften gegen die Abschaffung der verfassten Studierendenschaft) aktiv und gründete mit anderen zusammen eine JusoHochschulgruppe. Die einzige Veranstaltungsform, von der ich das Gefühl hatte, richtig zu profitieren, waren Seminare. Mein Studium bestand daher fast ausschließlich aus Seminaren, insgesamt vierzehn - überwiegend im Verfassungsrecht bei den Professoren Böckenförde und Hesse. Diese Seminare waren einfach großartig. Die beiden Professoren in ihrer persönlichen wie theoretischen Unterschiedlichkeit im engen Kreise zu erleben, mit ihnen über die Grundfragen des Verfassungsrechts zu diskutieren, waren echte Highlights. Von beiden habe ich viel gelernt.

Im Studium entdeckte ich auch die Frauenbewegung; mein erstes Buch: „Die Scham ist vorbei“ von Anja Meulenbelt. Wir gründeten eine Juristinnen-Gruppe, lasen zusammen Bücher, diskutierten und demonstrierten. Am Fachbereich gab es keine einzige Professorin, ich kämpfte um die Anrede „Frau“ statt „Fräulein“ (deutlich erschwert dadurch, dass meine Kollegin vom RCDS (Ring Christlich-Demokratischer Studenten) auf der Anrede „Fräulein“ bestand) und um Scheine, die mir nicht als „Herr stud. iur. Ute Sacksofsky“ überreicht wurden (führte zu großem Aufruhr im Fachbereichsrat).

Nach dem ersten Staatsexamen, das ich ohne Repetitor in einer Examensgruppe mit zwei Kommilitonen zusammen vorbereitet hatte, ging ich für zwei Jahre in die USA nach Harvard. Ich hatte ein McCloy-Stipendium erhalten und erwarb den Grad eines Master in Public Administration an der Kennedy School of Government. Die Zeit in den USA war phantastisch. Nie hatte ich mich so frei gefühlt, so viel anderes ausprobiert, so viel Neues kennengelernt und die Zeit einfach genossen. Inhaltlich hatte ich nach dem Ersten Staatsexamen genug von Jura und belegte daher vor allem Kurse in Informatik, Philosophie, in Verwaltungswissenschaften und Ökonomie (wobei mich die Spieltheorie schon wegen ihres Namens begeisterte). Lange stand auf der Kippe, ob ich in den USA bleiben würde (ich hatte mich in das Land und einen Amerikaner verliebt), jedenfalls aber den Referendardienst wollte ich zuerst noch in Deutschland absolvieren.

Zunächst aber stand die Zeit der Promotion an. Ich schwankte heftig zwischen zwei Themen, die mir beide am 